\title{
Strengthening Measures of Centralized Control Operation of Thermal Power Plant
}

\author{
Shiyang Bai, Hanwei Fu,Yanqi Zhong, Shaowei Wang \\ Thermal power plant, Petrochina liaoyang petrochemical company. Liaoyang, China \\ ardcbsy@petrochina.com.cn, blyguo1981@163.com
}

Keywords: power plant, centralized control operation, problem

\begin{abstract}
With the rapid development of science and technology, more and more advanced technology can be applied in power plant, include centralized control system. The automation level of power plant can be continuously improved by application of centralized control operation technology, which played a very important role in saving resources and decreasing energy consumption. However, as the application of centralized control operation, some problems were also increasingly appeared, in order to ensure the safe operation of power plant, scientific measures need to be formulated.
\end{abstract}

\section{Introduction}

Centralized control are comparative to independent control system. In comparison to mother pipe norm of older thermal power plant, centralized control technology is used by large medium power plant, namely unit system unit, a generator with a steam turbine and a boiler and the furnace, electric, machine are centralized to manage. The furnace, the electric and the machine are operated in the distributed control system (DCS) and each set of units is separated, which is defined as the centralized control operation. Centralized control operation is mainly production, operation and equipment outages, put forward the security measures to layout of maintenance and not responsible for the equipment maintenance and repair. A value of centralized control operation are generally equipped with a shift supervisor, one of the main and auxiliary on duty, head of a machine and a patrol to control and moniter the work of the unit. Arranging the relevant duty officer to monitor the production and operation of the centralized control machine for 24 hours, but because of the high degree of automation of centralized control operation, some power plants does not have this post.

\section{Technology of centralized control operation in power plant}

Main premise of centralized control operation, which is a new type of integrated control system, also known as DCS, is the automation of large and medium scale industrial production operation. Compared with the traditional technology, centralized control operation technology can fully reflect the integration, digitalization and high automation of modern industrial production. Control and management technology of production line in power plant is the main core of centralized control operation technology. Control technology of plant production line is make full use the function of the computer to further improve the automation level of power plant production, including the use of 4C technology on medium-sized continuous production line for automatic real-time monitoring technology, as well as manual operation control technique in unusual circumstances. Technical management is mainly responsible for improve the work efficiency of power plant, including the use of 4C technology on statistical analysis of information, scheduling of centralized control operation process and optimizing and guiding the economic operation v preventing and controlling production of production accident. 


\section{Measures to strengthen centralized control operation of power plant}

\subsection{Scientific and reasonable control measures}

(1) Increasing the application of advanced technology. The current substation begin to develop in the direction of unmanned on duty, with the rapid development of information technology, reliability of centralized control operation and monitoring system are improved, centralized control of signal is realized, the workload of the monitoring and operator on duty are reduced and the remote automatic meter reading is realized, which reduce the workload of equipment outage and maintenance, and make the equipment more reliable. Unattended substations will continue to develop standardization and scale, in which it is needed to improve efficiency of centralized control operation and enhance the enthusiasm of the staff, so as to realize the unattended substation.

(2) The key problems of pay attention to centralized control operation in power plant. At prevent, most of power plants still follow the traditional equipment, resulting to the working interface blank and low sharing base. But in the current power plant, whether it is the total amount of power transmission and distribution, or the total substation are to a certain extent increased, so the current management model is unable to meet the needs of the development and some drawbacks will continue to be exposed. Therefore, in this case, the power plant needs to optimize the allocation of resources, so that more rational allocation of technology, improve the professional level of front-line staff and improve the management system of power plants. But owing to different situation of each power plants, so for actual situation of their own power plant, it is necessary to plan to promote process reengineering and improve professional differentiation degree and professional quality in a certain extent, so as to realize the resource sharing and "streamlined production".

\subsection{Improve the environmental conditions of the centralized control system}

The external environment of centralized control system include: environmental requirements for electronic and control room, computer control system grounding, uninterruptible power supply and instrument air supply, safe and stable operation of the control system are directly affected by the state of these devices. In the process of operation, the centralized control system needs to have uninterrupted power supply, grounding system, to ensure that the control room and the electronic room to meet the corresponding requirements of the environment. In the unit installation and commissioning process, due to the tight schedule and heavy task, these devices are easily ignored. It is so vulnerable to outside interference that need good grounding system and cable shielding. The air conditioning system of control room and electronic room should be separated, because only one set of air conditioning system are used by electronic and control room commonly, and air conditioning of electronic room only regulate temperature, such in the air humidity of the season, the module is easy to condensation and in the dry season, static electricity is easy to happen. Whether supply mode and the switching time of UPS power are meet the requirements. The gas pipe of pneumatic actuator is exposed outside and freeze easily, leading to the actuator happen maloperation phenomenon. In the running process of the centralized control system, staff should pay particular attention to the problems, a nip in the bud, avoid unnecessary fault interruptting the normal operation of the thermal power plant.

\subsection{Strengthen the operation and management of centralized control system}

(1) The centralized control system is composed of software, hardware and disk station equipment of DCS system and transmitter, measuring switch, cable and actuator. Any equipment failure will cause paralysis of the system, loss of the function or cause the system failure, meanwhile may lead to damage and unit trip. So in the daily work, these centralized control device need to be regard as a whole to carry on the management and maintenance, making the site management and management of computer system maintenance working at the same time, so as to ensure stability of the control system.

(2) Centralized control system consists in hardware and software, which not only has a high reliability, large storage capacity and strong real-time, a variety of complex control strategy can be carried out by software in the running process, hardware and software need to be in the same 
position in the management of maintenance work. Currently many power plants tend to pay more attention to maintenance and management work of the hardware, and ignore software, which leads to the software configuration could be revised by everyone and cannot timely to copy and backup timely, thus leaving greater security risks.

(3) In order to assure the safety of operation units and personal control, factory set the engine protection system can timely stop calculation and protect the equipment from damage in abnormal operation of the unit. Safety protection and limit protection value of thermal protection system can be set to protect device, so as not to arbitrarily change and shall be temporary changed after approved. Once the unit is abnormal, engine protection system will shutdown protection, so as to avoid accident expanding, generating safety of units and personnel and reduce the incidence of accidents effectively, so in addition to the special nature, the the equipment protection cannot be changed or cancel by the operator randomly.

\section{Conclusion}

Current under the drive of science and technology, the automation level of power plant are improved constantly. So on this basis, to strengthen the centralized control operation of power plant, it is inevitable that take scientific and reasonable measures and use advanced computer system, control strategies to achieve energy conservation of power plant, reduction of investment cost, reasonable configuration of resource, thus improve the operation of the security, stability and economy of power plant.

\section{References}

[1]. Y. Zhao, X. C. Pan, F. Wang. Centralized control operation technology of thermal power plant[J]. Public Communication of Science \& Technology, 7(2013) 45-46

[2]. X. X. Yang. The Development and trend of automatic control technology on thermal power plant[J]. China Instrumentation, 1(2001) 4-8

[3]. Y. T. Guo. The solution of thermal power automation[J]. China Instrumentation, 10(2009) 75-78

[4]. J. M. Qu. Analysis of centralized control operation technology of generating units in thermal power plant[J]. Science \& Technology Association Forum, 12(2012) 53-54 